\title{
ÉdITORIAL
}

\section{Formation en médecine d'urgence au Canada : les années passent, mais la question demeure}

\author{
Riyad B. Abu-Laban, MD, MScS
}

VoIr ARTICLE CONNEXE À LA PAGE 111

ENGLISH VERSION ON PAGE 101

$\mathrm{E}^{n}$ 1987, lors d'une entrevue d'admission à une résidence, je me suis retrouvé devant une situation difficile où je devais répondre à une question primordiale. On m'avait demandé quelle était la différence entre le programme postdoctoral en médecine d'urgence $[\mathrm{FRCP}(\mathrm{MU})] \mathrm{du}$ Collège royal des médecins et chirurgiens du Canada (CRMCC) et le CMFC(MU), programme du Collège des médecins de famille du Canada (CMFC). Mon hésitation traduisait mon incapacité à expliquer la différence entre ces deux programmes menant à un certificat en médecine d'urgence au Canada. Après cette pénible entrevue, j'ai travaillé plusieurs années à temps plein dans des salles d'urgence comme généraliste. Puis, j'ai fait ma résidence en médecine d'urgence dans le cadre du programme accrédité par le CRMCC et ai obtenu une maîtrise en épidémiologie. Vingt ans plus tard, je suis chercheur et médecin d'urgence à l'Hôpital général de Vancouver, un centre de traumatologie tertiaire et un hôpital de base qui offre le programme de résidence en médecine d'urgence du CRMCC. Pendant la plupart de mes quarts de travail à l'urgence, j'enseigne aux étudiants en médecine, et il arrive parfois que ceux et celles que la médecine d'urgence intéresse me posent la même question que je me posais en 1987. Eh bien ... j'ai toujours de la difficulté à répondre clairement à cette question, et encore moins avec conviction. Le temps est peutêtre venu de me demander pourquoi.

Le Canada est le seul pays qui offre deux programmes de certificat en médecine d'urgence agréés par deux collèges. Est-ce une des raisons pour lesquelles il est difficile de répondre à cette question fondamentale sur notre système? Les deux collèges ont des objectifs précis, et les deux programmes d'études semblent être compatibles avec ces objectifs. Mais est-ce que deux programmes de formation gérés indépendamment sont la solution idéale au Canada? Est-ce qu'ils préparent les médecins aux aspirations distinctes vers différentes carrières? Tout porte à croire que la réponse à ces questions essentielles est un « non » catégorique. À mon sens, les rares ressources humaines et didactiques à leur disposition sont mal employées en raison des incohérences, des redondances et des inefficacités du système actuel. Qui plus est, j'estime que cette voix divisée, résultant de ces deux démarches vers l'obtention d'un certificat, est devenue un obstacle grandissant au développement de notre spécialité et à notre force politique. Or, nous demeurons paralysés face à la possibilité de la réforme de notre système actuel à deux volets, même si son évolution et sa continuité ont plus à voir avec les politiques et les forces du marché qu'avec une vision ou des normes.

L'historique de nos deux programmes de certificat est bien documenté ${ }^{1-3}$, mais le questionnement sur cette bizarre approche canadienne à la formation en médecine d'urgence ne date pas d'hier. En effet, au temps du Communiqué (bulletin qui a précédé le $J C M U$ ) de l'Association canadienne des médecins d'urgence (ACMU), les questions fondamentales relatives à la formation et au certificat en médecine d'urgence ont donné lieu à des débats vigoureux. ${ }^{4-9}$ En 1998, l'ACMU a formé un groupe de travail afin d'étudier cette question. ${ }^{10}$ Toutefois, la non-atteinte d'un consensus par le passé ne devrait pas nous empêcher d'examiner de nouveau la question. La médecine d'urgence diffère considérablement de ce qu'elle était il y a 10 ans. Notre discipline est désormais fermement établie et reconnue. Plusieurs universités canadiennes ont des

Du département de médecine d'urgence, Hôpital général de Vancouver, Vancouver (C.-B.)

Les opinions exprimées dans les éditoriaux sont celles des auteurs; elles ne reflètent pas nécessairement celles du JCMU ou de l'Association canadienne des médecins d'urgence.

JCMU 2008;10(2):104-7 
départements de médecine d'urgence, et la formation en médecine d'urgence fait maintenant partie de la rotation de base dans la majeure partie des écoles de médecine. Les soins d'urgence au Canada continueront certes d'être fournis par des médecins de famille n'ayant pas de certificat en MU ou de formation officielle en la matière. Il n'en demeure pas moins que l'obtention d'un certificat en MU est la norme des nouveaux médecins qui ont l'intention de poursuivre une carrière à temps plein en médecine d'urgence. Les postes dans la majeure partie des centres hospitaliers universitaires et des grands centres exigent maintenant un certificat quelconque en MU. Cette exigence ne provient pas d'une autojustification, mais bien d'un besoin clinique grandissant de traiter précocement les patients.

La terminologie que nous employons est au centre des débats concernant la médecine d'urgence au Canada. Nous avons évité de définir des termes tels que "prestation de soins d'urgence », «médecin d'urgence », et «spécialiste en médecine d'urgence ». À ce jour, l'ACMU a naturellement adopté une approche inclusive et, en dépit de son nom, se définit comme la « voix nationale en médecine urgence » (plutôt que la voie nationale des médecins urgence). ${ }^{11}$ Quand une spécialité en est à ses balbutiements, il est normal d'éviter les distinctions inutiles. Toutefois, le programme de certificat en MU existe depuis plus d'un quart de siècle au Canada, et il importe d'utiliser une terminologie claire si l'on veut un jour s'exprimer d'une voix unique et souscrire à des normes bien définies. Il est plus que temps de reconnaître le fait que les praticiens qui soignent les patients dans les urgences ne sont pas tous des urgentologues, comme ceux et celles qui font des accouchements ne sont pas tous des obstétriciens. Il est indéniable qu'au Canada, des médecins ayant diverses compétences professionnelles fournissent, quotidiennement, d'excellents soins d'urgence. ${ }^{12}$ Cependant, dans toutes les disciplines établies de la médecine, le nom d'une spécialité doit éventuellement devenir synonyme d'agrément. Un cardiologue, par exemple, est par définition un médecin titulaire d'un certificat en cardiologie. N'en sommes-nous pas au point où notre objectif devrait être la prestation de soins optimaux, dans la majorité des services d'urgence, par des médecins d'urgence agréés? L'American College of Emergency Physicians a maintenu cette position pendant presque deux décennies. L'ACMU ne devrait-elle donc pas épouser cette cause et fixer un objectif national à atteindre dans un délai bien précis?

Bien que bon nombre de médecins (y compris moimême dans mes années prérésidence) aient acquis leurs connaissances en médecine d'urgence « sur le tas » et sans formation officielle, une telle méthode est loin d'être idéale. Notre silence collectif sur la question du certificat en médecine d'urgence constitue une approbation tacite à l'égard des futurs médecins d'urgence qui acquerront leurs connaissances de la même manière. Certains diront que nous n'avons pas les ressources nécessaires pour former suffisamment de médecins d'urgence pour répondre aux besoins futurs au Canada. Plutôt que d'être une raison d'éviter d'adopter des normes appropriées, cette affirmation ne devrait-elle pas être une raison de défendre vigoureusement la cause pour obtenir plus de créneaux de formation (notion qu'une norme plus appropriée faciliterait). Dans sa soumission à la commission Romanow en 2002, l'ACMU avait demandé d'exiger « des compétences de base minimales pour tous les professionnels de la santé » dans les salles d'urgence au Canada. ${ }^{13}$ De telles compétences pourraient être définies en avalisant un programme de certificat en MU. (La sanction d'un programme de certificat en MU est le moyen approprié de définir de telles compétences.)

Quant au terme «spécialiste en médecine d'urgence », il est susceptible d'entraîner la discorde et, à mon avis, si l'ACMU tarde à agir à titre de la société spécialisée dont nous avons de plus en plus besoin, c'est certainement en grande partie dû à ce facteur. Contrairement au programme du CRMCC, la formation en MU offerte par le CMFC était destinée à améliorer la prestation de soins d'urgence par les médecins de famille en leur proposant une formation complémentaire en MU et non à créer des médecins d'urgence de carrière..$^{1,14}$ Or, les études ont révélé que la grande majorité des diplômés ayant obtenu leur CMFC(MU) pratiquent la médecine d'urgence à temps plein et qu'ils sont peu nombreux à pratiquer la médecine familiale. ${ }^{15,16}$ Bon nombre des plus éminents cliniciens, enseignants, chercheurs et administrateurs œuvrant en MU sont titulaires d'un certificat CMFC(MU). Ce sont de véritables «spécialistes » des urgences dans tous les sens du mot sauf le sens technique, car les collèges provinciaux réservent cette appellation aux titulaires de la désignation FRCP. Qui plus est, bien que les médecins titulaires du certificat CMFC(MU) constituent la majorité des médecins d'urgence agréés, ils sont représentés par un Collège dont l'objectif premier est la médecine familiale et qui n'est pas mandaté pour former des spécialistes en MU. Il n'est donc pas surprenant que certains médecins titulaires du certificat CMFC(MU) aient dit se sentir comme des « citoyens de seconde zone $»^{17}$, une situation malheureuse qui met en évidence la division actuelle au sein de notre spécialité. Parallèlement, les suggestions selon lesquelles les postes exigeant au minimum un certificat du CRMCC sont des postes « élitistes » et la conviction, par certains, qu'après un 
nombre arbitraire d'années, tout avantage provenant de leur formation additionnelle est annulé sont, à mon avis, deux points qui préoccupent grandement bon nombre des médecins d'urgence ayant reçu leur formation du CRMCC.

Les partisans de tels concepts proposent souvent, de façon rétrograde, qu'en dépit du programme d'enseignement normalisé et de l'évaluation validée que la spécialité a établis, les candidats devraient être jugés individuellement, strictement sur des mérites subjectifs, plutôt que d'avoir à surmonter les obstacles des titres de compétence. De toute évidence, les diplômés des deux programmes d'études ressentent des frustrations avec le système actuel.

Les ressources existantes pour former les médecins d'urgence au Canada sont inadéquates. ${ }^{13,18,19}$ Pour utiliser au mieux ces ressources, nous devons chercher à faire correspondre l'expérience de formation de chaque résident à la pratique visée. Les ressources sont mal affectées quand un futur médecin d'urgence inscrit au programme CMFC(MU) passe les deux premières années de sa formation à perfectionner ses connaissances en médecine de famille pratiquée dans un bureau. Ce sont là des connaissances qu'il ou elle n'utilisera probablement jamais, aux dépens d'un programme d'études conçu soigneusement pour une carrière en MU. Entre-temps, on vente les mérites du programme du CRMCC qui s'échelonne sur plusieurs années supplémentaires, le présentant comme une avenue vers l'acquisition de connaissances non cliniques en MU, car il ne faut pas cinq ans pour former d'excellents médecins d'urgence. ${ }^{2}$ Pourtant, bon nombre de résidents au programme du CRMCC ne profitent pas de cette occasion. Ainsi, quand un futur médecin d'urgence inscrit au programme du CRMCC obtient son diplôme, ayant acquis des connaissances non cliniques qu'il ne met pas en pratique, nous sommes devant un autre exemple de mauvaise affectation des ressources.

À mon sens, il est plus que temps de définir une spécialité en médecine d'urgence forte et unifiée et de lui affecter adéquatement des ressources, optimisant ainsi la prestation de soins dans les urgences pour les Canadiens et les Canadiennes. Or, pour atteindre cet objectif, il faut du leadership qui, selon moi, doit venir de l'ACMU. Il serait bon de rétablir un groupe de travail pour examiner l'historique d'initiatives passées de l'ACMU dans ce domaine, procéder à des consultations à grande échelle, rassembler les intervenants clés et négocier une solution acceptable. Les deux collèges doivent être prêts à faire des compromis, à mettre de côté leurs intérêts personnels et à œuvrer conjointement au renouvellement du système, en fondant leurs efforts sur les principes. Durant ce processus, je suggère que l'on réfléchisse aux sages paroles de Grant Innes, le premier rédacteur en chef du JCMU. En 2002, il a rédigé un éditorial percutant dans lequel il dressait une longue liste des compétences que les médecins d'urgence doivent posséder et déclarait que les médecins de famille et les médecins d'urgence ne sont ni identiques, ni interchangeables. ${ }^{20}$ Dans ce même ordre d'idées et en dépit du fait que la médecine familiale et la médecine d'urgence se chevaucheront toujours et auront toujours des liens communs, il n'est pas logique que les médecins d'urgence reçoivent leur formation et soient agréés par le Collège des médecins de famille du Canada. De même, il n'est pas logique de créer un collège de médecine d'urgence distinct ou une bourse de recherche de l'ACMU, comme l'ont proposé certains. ${ }^{6,16}$ Le Collège royal des médecins et chirurgiens du Canada a été créé en 1929 en vertu d'une loi spéciale du Parlement du Canada, afin d'encadrer la formation médicale des spécialistes au Canada, ${ }^{21}$ et la MU a fait d'innombrables progrès en s'affiliant au CRMCC. C'est au sein du Collège royal que la réforme et la coordination d'un programme de formation en MU et son agrément devraient résider. La solution que je propose est semblable à celle que plusieurs personnes ont faite précédemment : un programme d'études commun et d'une longueur appropriée (trois à quatre ans tout au plus) menant à une spécialisation en MU clinique, suivi d'une option d'une ou deux années supplémentaires pour ceux et celles qu'une carrière universitaire intéresse. ${ }^{1,22}$ Il faudrait également examiner soigneusement le pour et le contre d'un étroit créneau d'admissibilité, par la voie de la pratique, aux examens du programme d'études commun. Il y a 10 ans, dans le premier numéro du $J C M U$, était publié un article intitulé Formation en médecine d'urgence au Canada. Les auteurs concluaient que «les citoyens et les citoyennes méritent un programme de formation en $\mathrm{MU}$ normalisé, agréé et accrédité, afin de former des médecins d'urgence de haut calibre $\gg .{ }^{1}$ Je suis tout à fait d'accord, et il est grand temps que nous passions aux actes.

Conflits d'intérêts : Le $\mathrm{D}^{\mathrm{r}}$ Riyad B. Abu-Laban est associé du Collège royal des médecins du Canada $[\mathrm{FRCP}(\mathrm{C})]$ et détient un certificat de spécialiste en médecine d'urgence du CRMCC. Il est professeur adjoint au département de chirurgie de l'Université de Colombie-Britannique.

Mots clés : médecine d'urgence, formation, certificat, résidence, normes

\section{Références}

1. Moore K, Lucky C. Emegency medicine training in Canada. CJEM 1999;1:51-3. 
2. Ducharme J. Preparing emergency physicians for the future. CMAJ 2003;168:1548-9.

3. Steiner IP. Emergency medicine practise and training in Canada. CMAJ 2003;168:1549-50.

4. Ducharme J, Innes G. The FRCPC vs CCFP(EM): Is there a difference 10 years after residency? CAEP Communiqué 1997;Fall:1-4.

5. The FRCPC vs CCFP(EM) [correspondance]. Voir l'échange de correspondance dans : CAEP Communiqué 1997-98;Winter:7-13.

6. Etherington J. An immodest proposal: the future of emergency medicine training in Canada. CAEP Communiqué 1997-98; Winter:16-9.

7. The FRCPC vs CCFP(EM) [correspondance]. Voir l'échange de correspondance dans : CAEP Communiqué 1998;Spring:7-9.

8. On unified EM training [correspondance]. Voir l'échange de correspondance dans : CAEP Communiqué 1998;Summer:7-11.

9. Gray S. Emergency medicine residency training [correspondance]. CAEP Communiqué 1998;Fall:5.

10. Grunfeld AF. The task force on emergency medicine training. CAEP Communiqué 1998;Summer:1-2.

11. About CAEP. Association canadienne des médecins d'urgence. Disponible sur : www.caep.ca (Consulté le 19 janvier 2008).

12. Lund A. I am emergency medicine [correspondance]. CJEM 2006;8:4-5.

13. Working CAEP. Group on the Future of Emergency Medicine in Canada. The future of emergency medicine in Canada: submission from CAEP to the Romanow Comission. Part1. CJEM 2002;4:359-68.
14. Examens-Certification. Le Collège royal des médecins et chirurgiens du Canada. Disponible sur : www.cfpc.ca/French /cfpc/home/default.asp?s=1 (Consulté le 19 janvier 2008).

15. Chan BT. Do family physicians with emergency medicine certification actually practise family medicine. CMAJ 2002;167:869-70.

16. Shepherd LG, Burden JK. A survey of one CCFP-EM program's graduates: their background, intended type of practice and actual practice. CJEM 2005;7:315-20.

17. Campbell S. CCFP-EM versus FRCP [correspondance]. CJEM 2003;5:80.

18. Steiner IP, Yoon PW, Holroyd BR. Manpower crisis in emergency medicine: Can residency programs make an impact? CJEM 2000;2:103-5.

19. Working CAEP. Group on the Future of Emergency Medicine in Canada. The future of emergency medicine in Canada: submission from CAEP to the Romanow Comission. Part2. CJEM 2002;4:431-8.

20. Innes G. Thinking outside the box. CAEP Working Group on the Future of Emergency Medicine in Canada. CJEM 2002;4:316.

21. À propos du CRMCC. Le Collège royal des médecins et chirurgiens du Canada. Disponible sur : http://crmcc.medical .org/ (Consulté le 19 janvier 2008).

22. MacDonald RD. How to train emergency docs. CMAJ 2003; 169:389.

Correspondance : $\mathrm{D}^{\mathrm{r}}$ Riyad Abu-Laban, Département de médecine d'urgence, Hôpital général de Vancouver, 855, 12e Avenue Ouest, Vancouver BC V5Z 1M9; abulaban@interchange.ubc.ca 